\title{
ENTUPIMENTO DE GOTEJADORES EM FUNÇÃO DA APLICAÇÃO DE SUPERFOSFATO SIMPLES E ÁCIDO NÍTRICO
}

\author{
ALLAN C. BARROS ${ }^{1}$, RUBENS D. COELHO ${ }^{2}$, PEDRO R. F. MEDEIROS ${ }^{3}$, \\ RALINI F. DE MELO ${ }^{4}$, CÍCERO R. A. BARBOZA JÚNIOR ${ }^{3}$, CARLOS T. DOS S. DIAS $^{5}$
}

RESUMO: O superfosfato simples (SS) é uma alternativa aos adubos fosfatados que são utilizados via fertirrigação, como o ácido fosfórico, que provoca acidificação do bulbo úmido no solo, e o monofosfato de amônio purificado, que apresenta maior preço. No entanto, não se conhece o efeito de tal adubo no funcionamento de emissores, principalmente nos gotejadores, nem se é possível controlar o entupimento por SS por meio de aplicações de ácido. Assim, desenvolveu-se este trabalho com o objetivo de avaliar a possibilidade da utilização do superfosfato simples via gotejamento, como alternativa ao uso de outros adubos fosfatados, e a tentativa de controle do entupimento utilizando ácido nítrico. O trabalho foi desenvolvido em bancada de ensaios de gotejadores, localizada no Laboratório de Hidráulica da ESALQ/USP. Foram aplicadas doses de 50; 75 e $100 \%$ da solubilidade do superfosfato simples (SS), em 26 tubogotejadores de diferentes marcas comerciais, e ao fim de 360 horas de irrigação, foi aplicado ácido nítrico com pH 2, na tentativa de desentupir os gotejadores mais sensíveis. Diante dos resultados, foi possível inferir que o uso do superfosfato simples pode ser feito via fertirrigação, e o ácido nítrico, aplicado segundo esta metodologia, não proporcionou a desobstrução dos emissores.

PALAVRAS-CHAVE: adubos fosfatados, gotejamento, obstrução de emissores.

\section{DYNAMIC BLOCKAGE OF DRIPPERS AS A FUNCTION OF THE APPLICATION OF SUPER SIMPLE PHOSPHATE AND NÍTRIC ACID IN LOCALIZED IRRIGATION}

\begin{abstract}
The super simple phosphate (SS) is an alternative to the phosphorous fertilizers which are used through fertigation, as the phosphoric acid, which triggers acidification of the wetted volume in the soil, and the monophosfato of purified ammonium, that has a higher price. However, the effect of such fertilizer in the emitters function, mainly in the drippers, is not known neither if it is possible to control the blockage for SS through acid applications. Thus, this study was developed in order to evaluate the possibility of the use of super simple phosphate trough drip irrigation, as an alternative to the use of other phosphorous fertilizers, and the attempt of controlling the blockage with nitric acid. The work was carried out in bench of drippers rehearsals, located at the Hydraulic laboratory in ESALQ-USP. Doses of 50; 75 and 100\% of the super simple phosphate (SS) were applied in 26 emitter tubes with different commercial marks, and after 360 hours the nitric acid with a $\mathrm{pH}$ of 2 was applied, in the attempt of clearing the most sensitive drippers to the blockage. With the results it was possible to infer that: the use of super simple phosphate can be done by fertigation without causing damages to the system and the nitric acid, applied according to this methodology, did not provide the clearance of the emitters.
\end{abstract}

KEYWORDS: drip irrigation, blockage of emitters, phosphorous fertilizer.

\footnotetext{
${ }^{1}$ Prof. Assistente, Curso de Agronomia, UFAL, Câmpus Arapiraca, Caixa Postal 61, Arapiraca - AL, allan-cunha@ @otmail.com

${ }^{2}$ Prof. Doutor, Departamento de Engenharia Rural, ESALQ/USP, Piracicaba - SP, rdcoelho@esalq.usp.br

${ }^{3}$ Doutorandos do curso de Irrigação e Drenagem, ESALQ/USP, Piracicaba - SP, prfmede@ esalq.usp.br, crabjuni@esalq.usp.br

${ }^{4}$ Doutora em Irrigação e Drenagem, ESALQ/USP, Piracicaba - SP, ralini@ @esalq.usp.br

${ }^{5}$ Prof. Doutor, Departamento de Ciências Exatas, ESALQ/USP, Piracicaba - SP, ctsdias@esalq.usp.br

Recebido pelo Conselho Editorial em: 24-4-2008
}

Aprovado pelo Conselho Editorial em: 15-4-2009 


\section{INTRODUÇÃO}

No Brasil, a utilização de fosfato na agricultura é prática comum tanto em cobertura como por meio da fertirrigação, e seu consumo é da ordem de $38.150 \mathrm{t}$ para o fosfato natural, $534.045 \mathrm{t}$ de fosfato monoamônico (MAP) e 934.887 t de superfosfato simples (ANUÁRIO ESTATÍSTICO, 2004).

Em razão da baixa solubilidade, tradicionalmente o fósforo é aplicado em fundação, antes do plantio. No entanto, segundo VITTI et al. (1994), nutrientes como fósforo, cálcio e magnésio, que em condições de sequeiro são mais retidos no solo, quando aplicados via fertirrigação, têm a movimentação através do perfil do solo acelerada, o que, em parte, ajuda a explicar os ganhos de eficiência com esse sistema podendo, por meio da irrigação por gotejamento, aumentar o movimento de fósforo de 5 a 10 vezes (VILLAS BÔAS et al., 1999).

Com a introdução no mercado de nutrientes fosfatados solúveis em água, como, por exemplo, o fosfato monoamônico (MAP) e o ácido fosfórico, partiu-se para o estudo da sua aplicação diretamente na água de irrigação (BRITO et al., 2000). BRITO et al. (1996) avaliaram o efeito de duas fontes de fósforo, superfosfato simples e MAP aplicadas convencionalmente e via água de irrigação, na cultura do melão, e não observaram diferenças significativas entre as fontes, quando aplicadas de modo convencional e/ou via água de irrigação por 30 e 42 dias após o plantio.

Geralmente, em irrigação localizada, é utilizado o monofosfato de amônio purificado, que, no entanto, possui maior preço (FORATTO et al., 2007), ou a utilização do ácido fosfórico, que pode ocasionar corrosão em peças metálicas dos sistemas de irrigação (VILLAS BÔAS et al., 1999), e sua constante aplicação pode ocasionar a acidificação do bulbo molhado do solo, afetando a solubilidade, a concentração em solução e a forma iônica dos nutrientes no solo e, consequentemente, a absorção e a utilização desses pela planta (MCBRIDE et al., 1997 citados por FAGERIA, 2000). Surge, assim, a possibilidade de avaliar o uso do superfosfato simples via fertirrigação.

Segundo FORATTO et al. (2007), a aplicação de fertilizantes fosfatados, tais como superfosfofato simples, superfosfofato triplo e monofosfato de amônio, via gotejamento, pode apresentar incrustações nas canalizações e obstruções de emissores, no entanto nenhum trabalho foi feito para confirmar essa afirmação, muito menos o controle do possível entupimento por meio da aplicação de ácidos.

$\mathrm{O}$ uso de ácidos diminui as obstruções provocadas por precipitados químicos, reduzindo o $\mathrm{pH}$ da água, aumentando a solubilidade desses elementos, mantendo-os em solução; essa prática tem sido recomendada por vários autores (TEIXEIRA, 2006), sendo o ácido clorídrico ( $\mathrm{HCl}) 12 \mathrm{~N}$, o ácido sulfúrico $\left(\mathrm{H}_{2} \mathrm{SO}_{4}\right) 36 \mathrm{~N}$, o ácido nítrico $\left(\mathrm{HNO}_{3}\right) 16 \mathrm{~N}$, utilizados por MEDEIROS et al. (2008), e o ácido fosfórico $\left(\mathrm{H}_{3} \mathrm{PO}_{4}\right) 45 \mathrm{~N}$ os mais utilizados para esse propósito (NAKAYAMA \& BUCKS, 1986).

Assim, o presente trabalho teve como objetivo avaliar a possibilidade do uso de superfosfato simples via fertirrigação por gotejamento, como alternativa ao uso de outros adubos fosfatados, e a tentativa de controle do entupimento de emissores utilizando ácido nítrico.

\section{MATÉRIAL E MÉTODOS}

O experimento foi conduzido no Laboratório de Irrigação, no Departamento de Engenharia Rural, da Escola Superior de Agricultura "Luiz de Queiroz" - ESALQ/USP, Piracicaba - SP, no período de setembro a novembro de 2006.

O delineamento estatístico utilizado foi o inteiramente aleatorizado, sendo cada emissor considerado como parcela experimental, e cada parcela repetida dez vezes. Foram utilizados 26 emissores de diferentes marcas comerciais encontradas no mercado: Amanco, Netafim, Azud, Toro, Plastro, Irrimon, Naandan, Petroísa, Queen Gil. A descrição de cada emissor pode ser encontrada na Tabela 1. 
Como os emissores ensaiados são produtos comerciais, os dados e as análises aqui apresentadas foram codificados para evitar especulação comercial dos resultados apresentados, uma vez que os ensaios conduzidos não são normatizados. Os números utilizados na codificação dos modelos de emissores (T1, T2, T3,...,T26) não têm nenhuma relação com a sequência dos fabricantes citados anteriormente, bem como a quantidade de emissores não é proporcional ao número de fabricantes. Contudo, a identificação parcial dos emissores poderá ser solicitada via e-mail aos autores.

TABELA 1. Fabricantes, emissores com relação ao fluxo, vazão, diâmetro nominal ( $\varnothing \mathrm{N})$ e distância entre emissores (DEG). Description of the drippers: manufacturers, flow, diameter $(\varnothing \mathrm{N})$ and distance between emitters (DBE).

\begin{tabular}{cccccc}
\hline Tratamento & Design & Fluxo & Vazão $\left(\mathrm{L} \mathrm{h}^{-1}\right)$ & $\emptyset \mathrm{N}(\mathrm{mm})$ & DEG $(\mathrm{cm})$ \\
\hline T1 & Fita & NC & 1,4 & 17 & 0,50 \\
T2 & Fita & NC & 2,0 & 17 & 0,80 \\
T3 & Fita & AC & 2,2 & 16 & 0,50 \\
T4 & Pastilha & AC & 2,5 & 17 & 0,75 \\
T5 & Pastilha & AC & 1,6 & 16 & 0,75 \\
T6 & Bob & NC & 2,0 & 16 & 0,80 \\
T7 & Bob & AC & 2,0 & 17 & 0,80 \\
T8 & Bob & AC & 2,2 & 17 & 0,80 \\
T9 & Bob & AC & 2,2 & $16 / 25$ & 0,75 \\
T10 & Bob & AC & 2,35 & $16 / 40$ & 0,80 \\
T11 & Bob & NC & 3,0 & 17 & 0,40 \\
T12 & Bob & AC & 2,5 & 16 & 1,00 \\
T13 & Pastilha & AC & 1,1 & 16,4 & 0,40 \\
T14 & Pastilha & NC & 2,0 & 16 & 0,75 \\
T15 & Bob & AC & 2,1 & 16 & 0,80 \\
T16 & Bob & AC & 3,8 & 16 & 0,90 \\
T17 & Bob & AC & 1,6 & 16 & 0,50 \\
T18 & Pastilha & NC & 1,5 & 16 & 0,30 \\
T19 & Fita & NC & 4,0 & 16,5 & 0,10 \\
T20 & Pastilha & AC & 2,3 & 17 & 0,50 \\
T21 & Pastilha & AC & 1,6 & 16 & 0,50 \\
T22 & Pastilha & NC & 2,0 & 16 & 0,60 \\
T23 & Pastilha & NC & 2,05 & - & 0,75 \\
T24 & Pastilha & AC & 1,8 & 17,5 & 1,00 \\
T25 & Pastilha & AC & 1,6 & 16 & 0,33 \\
T26 & Pastilha & AC & 3,6 & 16 & 1,00 \\
\hline
\end{tabular}

* AC e NC - Autocompensante e Não compensante.
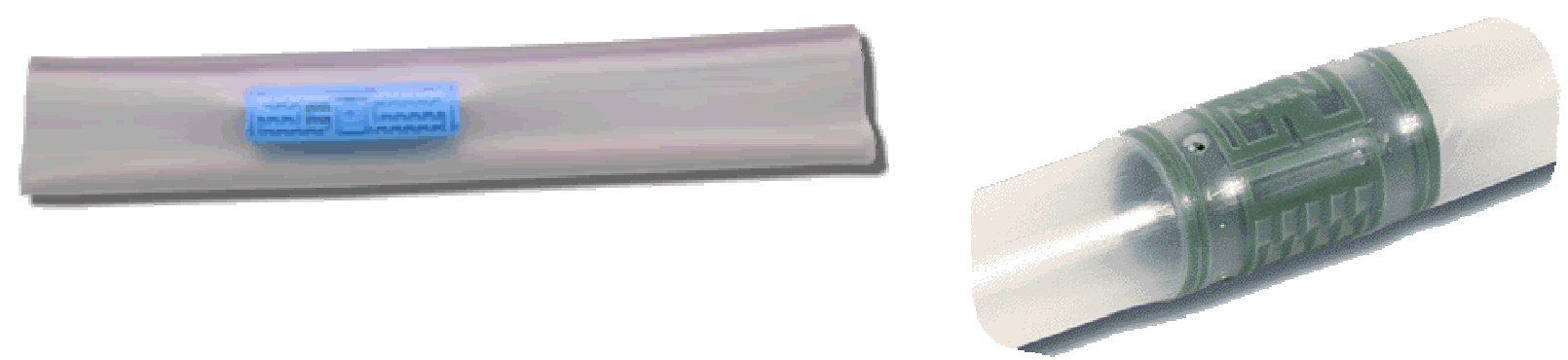

FIGURA 1. Design dos gotejadores tipo pastilha (lado esquerdo) e tipo bob (lado direito) (RETORRE NETO, 2008). Kind of drippers design (RETORRE NETO, 2008). 
O experimento foi realizado em bancada de estrutura metálica, composta por três andares, com 11,0 m de comprimento, 4,0 m de largura e 5,8 $\mathrm{m}$ de altura. Os ensaios foram conduzidos em uma das partes do primeiro andar, que é composto por sete níveis, com quatro linhas por nível, dispostas de forma aleatória.

Como não existe nenhuma norma técnica para esse tipo de ensaio, para avaliar a influência do superfosfato simples nos gotejadores, foi inicialmente aplicada água enriquecida, na concentração de $50 \%$ da solubilidade, e em seguida 75 e $100 \%$. As concentrações de 50 e $75 \%$ foram aplicadas num total de 72 horas, e a de $100 \%$ num total de 216 horas, sendo as soluções preparadas em tanque de $1.000 \mathrm{~L}$ e homogeneizadas. Toda água aplicada retornava ao tanque, por meio de calhas, formando um sistema com recirculação. A aplicação da solução de superfosfato simples tinha período de 12 horas, em intervalos de 36 horas, e após 7 dias era trocada a solução do tanque.

A pressão de serviço utilizada foi de $0,147 \mathrm{MPa}$, e a filtração da solução era feita em filtro de tela (130 mesh) para reter as partículas não dissolvidas na solução. A água de abastecimento do laboratório utilizada no ensaio provém de sistema de abastecimento da ESALQ/USP. Foi feito o monitoramento dessa água com a realização de análises químicas (Tabela 2).

TABELA 2. Resultado da análise química da água do laboratório de irrigação - ESALQ/USP. Result of chemical analysis of water for irrigation from the laboratory of ESALQ/USP, Piracicaba - SP, Brazil.

\begin{tabular}{lcc}
\hline Parâmetro & Unidade & Resultado \\
\hline Alcalinidade $\left(2 \mathrm{CO}_{3}\right)^{2-}+\mathrm{HC}^{3-}$ & $\mathrm{mg} \mathrm{L}^{-1}$ & 49,0 \\
Cloreto $\left(\mathrm{Cl}^{-}\right)$ & $\mathrm{mg} \mathrm{L}^{-1}$ & 30,5 \\
Sódio $\left(\mathrm{Na}^{+}\right)$ & $\mathrm{mg} \mathrm{L}^{-1}$ & 37,0 \\
Cálcio $\left(\mathrm{Ca}^{2+}\right)$ & $\mathrm{mg} \mathrm{L}^{-1}$ & 66,1 \\
Magnésio $\left(\mathrm{Mg}^{2+}\right)$ & $\mathrm{mg} \mathrm{L}^{-1}$ & 4,3 \\
Ferro $(\mathrm{Fe})$ & $\mathrm{mg} \mathrm{L}^{-1}$ & 0,08 \\
Condutividade elétrica $(\mathrm{CE})$ & $\mathrm{mS} \mathrm{cm}^{-1}$ & 0,40 \\
pH & & 7,4 \\
Gás carbônico $\left(\mathrm{CO}_{2}\right)$ & $\mathrm{mg} \mathrm{L}^{-1}$ & 3,1 \\
Acidez $(\mathrm{CaCO})$ & $\mathrm{mg} \mathrm{L}_{3}^{-1}$ & 8,2 \\
Dureza total $\left(\mathrm{CaCO}_{3}\right)^{*}$ & $\mathrm{mg} \mathrm{L}^{-1}$ & 182,7 \\
\hline
\end{tabular}

*Dureza total calculada com base no equivalente de carbonato de cálcio $\left(\mathrm{CaCO}_{3}\right)$, segundo FRANSON (1995): $2,497\left[\mathrm{Mg}, \mathrm{mg} \mathrm{L}^{-1}\right]$.

As vazões dos gotejadores foram determinadas por bateria de pluviômetros de massa conhecida, dispostos abaixo dos emissores, que retinham a água durante o período de 5 min, sendo os pesos medidos com balança eletrônica (precisão 0,001 g). As leituras eram efetuadas em intervalos semanais (até a sexta leitura), e a partir dessa, em intervalos quinzenais, continuando a aplicação de SS a 100\% da solubilidade.

Ao efetuar a medição, cada gotejador era cuidadosamente observado, no sentido de não haver interferência de vazões provenientes de gotejadores adjacentes e/ou de pontos de conexão; para tanto, barbantes foram utilizados para isolar a vazão de cada gotejador. Como as linhas de gotejadores possuem diferentes distâncias entre gotejadores (DEG, Tabela 1), para que houvesse a mesma quantidade de emissores avaliados, foram selecionados 10 emissores em cada linha, constituindo-se, assim, repetições. Esses eram marcados e mantidos durante o experimento.

De posse desses valores, foram feitos os seguintes cálculos:

$$
\mathrm{UD}=\frac{\mathrm{q} 25 \%}{\text { qmédia }} 100
$$


em que,

UD - uniformidade de distribuição, \%;

$\mathrm{q}_{25 \%}$ - vazão média de $1 / 4$ dos menores valores, $\mathrm{L} \mathrm{h}^{-1}$, e

$\mathrm{q}_{\text {média }}$ - média geral das vazões, $\mathrm{L} \mathrm{h}^{-1}$.

Qr $=\frac{\text { Qi }}{\text { Qa }} 100$

em que,

Qr - vazão relativa, \%;

Qi - vazão do início do experimento, $\mathrm{L} \mathrm{h}^{-1}$, e

$\mathrm{Qa}$ - vazão atual, $\mathrm{L} \mathrm{h}^{-1}$.

$\mathrm{GE}=\left(1-\frac{\mathrm{Qi}}{\mathrm{Qa}}\right) 100$

em que,

GE - grau de entupimento, $\%$.

C.V. $=\frac{\mathrm{S}}{\text { qmédia }} 100$

em que,

C.V. - coeficiente de variação, \%;

$\mathrm{S}$ - desvio-padrão, e

qmédia - vazão média dos emissores coletados, $\mathrm{L} \mathrm{h}^{-1}$.

Depois de transcorridas 360 horas de aplicação do SS, foi dado início à aplicação de ácido nítrico para desentupimento: esse procedimento foi adotado para todos os emissores, mesmo para aqueles que não foram sensíveis ao superfosfato simples. Para a determinação da quantidade de ácido a ser adicionada à água de irrigação, fez-se uma titulação e o pH aplicado foi de 2.

Após a estabilização da pressão e vazão, havia aplicação do ácido durante 10 min. Seguida da aplicação, as linhas que receberam o tratamento eram deixadas em repouso por 1 hora e depois lavadas com água do laboratório, mantendo essa aplicação de água do laboratório durante 24 horas. Após quatro aplicações consecutivas de ácido, foi realizada a última leitura.

\section{RESULTADOS E DISCUSSÃO}

Na Tabela 3, são apresentados os coeficientes de variação (C.V.) e a vazão relativa (Qr) dos emissores em função do tempo de aplicação do superfosfato simples (SS) e do ácido nítrico. Notase que, no momento zero, antes de ser iniciada a aplicação do produto, alguns gotejadores (T1, T6, T11, T15, T16, T21 e T23) já apresentavam C.V. acima de $10 \%$ e, desses, apenas o emissor T21 era desprovido de membrana de autocompensação. Em oposição, o emissor T2 já apresentava valor baixo de C.V. $(1,1 \%)$.

Após 72 horas de aplicação de SS (50\% da solubilidade), houve variação em todos os emissores, mostrando a suscetibilidade à aplicação do produto, com exceção de T3 e T5, que mantiveram a vazão. Nos emissores T1 e T16, a vazão foi reduzida em quase 55 e 75\%, já no T23 e no T24, a vazão teve aumento bastante significativo, 148 e $262 \%$, respectivamente. Os outros emissores mantiveram-se entre $90 \%$ e $114 \%$. O C.V., em relação ao tempo zero, variou e mostrou a recuperação dos emissores T6 e T11 e grande elevação no T24, superando os $100 \%$ de C.V., seguido pelo T6, que também não demonstrou bom resultado. $\mathrm{O}$ emissor que demonstrou o melhor resultado foi o T5 (C.V. 1,7\% e Qr 99,4\%).

Após 144 horas de aplicação de SS (75\% da solubilidade), os melhores valores de vazão relativa foram obtidos pelos emissores T4, T11 e T12 (desses, apenas o T12 não é autocompensante - AC), demonstrando, até o momento, serem menos influenciados pelo SS; o pior valor foi 
demonstrado pelo T1 (AC). O T24 tem destaque nessa avaliação por apresentar valor 77 pontos percentuais abaixo do valor há 72 horas. Vinte e um emissores mantiveram o Qr entre 90 e $100 \%$ e 16 apresentaram C.V. abaixo de $10 \%$, sendo os três melhores valores de C.V. para emissores não compensantes (NC).

Ao final da aplicação de 216 horas de SS à concentração de 100\%, a quantidade de emissores que manteve a variação da Qr e o C.V. acima de $10 \%$ caiu para $18 \%$ e $15 \%$, respectivamente. Os emissores que se mostraram mais sensíveis foram: AC: T1, T3, T11, T13, T16, T17 e T23, e NC: T12, T19, T22, T24; apesar de a maioria ser $\mathrm{AC}$, os dados não permitem inferir que a suscetibilidade ao SS (a 100\% da solubilidade) é afetada pelo fluxo, já que esses emissores representam 41,2\% dos AC e 44,4\% dos NC avaliados; assim, a sensibilidade em relação ao fluxo é aproximada. Os melhores valores de Qr foram para os emissores T9 e T10, e os piores para T1 e T23, todos AC. O melhor valor de C.V. foi apresentado pelo NC T2, que, apesar da elevação após a primeira fase (50\% do SS), foi recuperando até atingir $2 \%$.

TABELA 3. Dados médios referentes aos Coeficientes de variação (C.V.), vazão relativa (Qr) em função da aplicação de superfosfato simples e ácido nítrico. Variation Coefficient (C.V.) and relative flow (Qr) of drippers submitted to the application of Super Simple Phosphate and nitric acid.

\begin{tabular}{|c|c|c|c|c|c|c|c|c|c|c|c|c|}
\hline \multirow{3}{*}{$\begin{array}{c}\text { Emissor } \\
\text { (Fluxo) }\end{array}$} & \multicolumn{12}{|c|}{ Tempo de aplicação de SS } \\
\hline & \multicolumn{2}{|c|}{0 hora } & \multicolumn{2}{|c|}{72 horas } & \multicolumn{2}{|c|}{144 horas } & \multicolumn{2}{|c|}{216 horas } & \multicolumn{2}{|c|}{360 horas } & \multicolumn{2}{|c|}{ Ácido } \\
\hline & $\begin{array}{l}\mathrm{Qr} \\
(\%)\end{array}$ & $\begin{array}{l}\text { C.V. } \\
(\%)\end{array}$ & $\begin{array}{l}\mathrm{Qr} \\
(\%)\end{array}$ & $\begin{array}{l}\text { C.V. } \\
(\%)\end{array}$ & $\begin{array}{l}\text { Qr } \\
(\%)\end{array}$ & $\begin{array}{l}\text { C.V. } \\
(\%)\end{array}$ & $\begin{array}{l}\text { Qr } \\
(\%)\end{array}$ & $\begin{array}{l}\text { C.V. } \\
(\%)\end{array}$ & $\begin{array}{l}\text { Qr } \\
(\%)\end{array}$ & $\begin{array}{l}\text { C.V. } \\
(\%)\end{array}$ & $\begin{array}{l}\mathrm{Qr} \\
(\%)\end{array}$ & $\begin{array}{l}\text { C.V. } \\
(\%)\end{array}$ \\
\hline & 100,0 & 27,2 & 56,7 & 27,1 & 58,7 & 35,4 & 58,4 & 30,6 & 21,8 & & 40,4 & 79,8 \\
\hline $2-\mathrm{NC}$ & 100,0 & 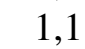 & 99,2 & 2 & 106,2 & 2,5 & 104,3 & 1,9 & 88,6 & & 100,9 & 3,8 \\
\hline &, 0 & & 0,0 & 1 & 4,7 & 11,3 & 08,5 & 11,9 & 84,9 & & 3,5 & 41,1 \\
\hline T4- & 0,0 & 3,6 & 104,2 & 5,3 & 00,7 & 4,9 & 110,1 & 7,2 & 03,2 & 4,7 & 1,4 & 6,5 \\
\hline T5 & 100,0 & 2,7 & 99,4 & 1,7 & 5,6 & 1,6 & 103,5 & 2,7 & 103,1 & 2,0 & 105,5 & 13,1 \\
\hline T6 & 100,0 & 36,0 & 4,8 & 6,7 & 09,3 & 4,9 & 109,7 & 3,6 & 109,4 & 4 & 0,6 & 15 \\
\hline & & & , & 14,4 & 1 & 3 & , & 4 & 98,6 & & 3 & \\
\hline & 0 & 9,3 &, 5 & 6 & 97,2 & 3,9 & 0 & 5,1 & 82,6 & & 5 & 10,6 \\
\hline T9- & 100,0 & 5,3 & 102,2 & 8,7 & 101,2 & 5,8 & 100,1 & 5,3 & 92,1 & 36,5 & 77,4 & 45,4 \\
\hline C & 0,0 & 2, & 97,5 & 3,4 & 98,9 & 2,7 & 99,4 & 2,7 & 100,7 & 3 & 93,6 & 5 , \\
\hline & 0 & 35 , & Y & & , & 11 & 97,4 & 10,5 & & & 2 & 11, \\
\hline & 0,0 & 0 & 94,3 & 13,9 & 100,6 & 2,7 & 84,9 & 29,4 & 94,9 & 13,8 & 90,2 & 7,8 \\
\hline $\mathrm{T} 13$ & 100,0 & 2 & 92,7 & 16,9 & 92,4 & 20,5 & 86,1 & 19,5 & 86,0 & 24,5 & 77,9 & 37,5 \\
\hline & 1 & 2 & 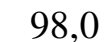 & 4,6 & 1 & 3 & 93,2 & 5, & 98,9 & 5 & 92,2 & 8 , \\
\hline & 0 & 12,1 & 1,4 & 13 & 9 & 5,6 & 99,1 & 3,8 & 94,0 & 26 & 9,2 & 20 \\
\hline & 0,0 & 13,8 & .2 & 44,5 & 81,1 & 34,1 & 81,8 & 35,7 & 96,0 & 37,7 & 78,0 & 37,3 \\
\hline T17-AC & 100,0 & 2 & 97,0 & 11,5 & 103,3 & 8,7 & 109,1 & 12,5 & 99,1 & 15,0 & 91,6 & 10,7 \\
\hline & & 3 & 102,0 & 4,6 & 4,2 & 5,2 & 0 & 5,3 & 7,1 & 11 & 94,8 & 7. \\
\hline &, 0 & 4 & 109,0 & 31,3 & 104,7 & 19,9 & 109,8 & 29,7 & 107,9 & 39,7 & 95,0 & 52,9 \\
\hline & 100,0 & 1,9 & & 2,6 & & & 95,7 & 3,5 & 86,4 & 5,2 & 88,3 & 4,5 \\
\hline $\mathrm{T} 21-\mathrm{NC}$ & 100,0 & 28,1 & 94,0 & 4,3 & 91,5 & 4,8 & 97,1 & 5,2 & 89,8 & 48,9 & 93,6 & 45,5 \\
\hline & 100,0 & & & 19,2 & 98,9 & 11,0 & 103,8 & 12,4 & 92,8 & 24,3 & 85,7 & 26,0 \\
\hline &, 0 & 31,8 & 148,3 & 7,9 & & 13,0 & 13 & 15,6 & 94,7 & 83,4 & 127,2 & 12,9 \\
\hline & 100,0 & & 262,1 & 04,5 & & 97,1 & 90,5 & 98,9 & 79,0 & 110,0 & 151,2 & 101,1 \\
\hline $\mathrm{T} 25-\mathrm{AC}$ & 100,0 & 2,5 & 102,8 & 3,4 & 109,2 & 5,5 & 114,6 & 8,8 & 111,8 & 6,6 & 111,6 & 3,7 \\
\hline T26-AC & 100,0 & 2,1 & 101,9 & 5,1 & 115,6 & 18,8 & 105,9 & 4,5 & 115,9 & 6,9 & 120,5 & 9,2 \\
\hline
\end{tabular}

A passagem de SS pelos gotejadores mostrou, ao final da análise, que alguns melhoraram suas condições iniciais; outros, apesar da sensibilidade ao primeiro contato, mantiveram seus valores de Qr e C.V., e os restantes se mostraram suscetíveis ao produto. Desse modo, o T1 apresentou valores 
baixos inicialmente e manteve a desuniformidade, semelhantemente ao T23, que iniciou com valores altos. Os emissores T2, T4, T5, T9 e T26 reagiram e mantiveram seus valores sempre abaixo de $10 \%$, semelhantemente ao emissor T3, embora esse não tenha conseguido manter seu C.V. de modo uniforme. Os T6, T8, T11, T15 e T21 foram beneficiados pela aplicação do SS para a redução do C.V., ao contrário dos emissores T7, T16, T17, T19, T20, T22, T25 e T18. Apesar disso, o emissor T18 manteve seus valores finais abaixo de 10\%. Os emissores T10 e T14 melhoraram suas Qrs após a aplicação do SS, embora seus C.Vs. tenham aumentado. Os T12 e T13 conseguiram manter suas Qrs até a segunda fase (75\% de SS), mas não resistiram ao aumento de concentração, fazendo com que sua Qr caísse. O T24 apresentou as maiores variações de Qr e C.V., finalizando com o Qr abaixo de 10\%; apesar disso, o C.V. foi de quase $100 \%$.

A partir dos dados, foi proposta a Tabela 4 para comparar a sensibilidade dos emissores à aplicação de superfosfato simples.

TABELA 4. Análise de sensibilidade de emissores em função da aplicação de superfosfato simples. Sensitivity analysis of drippers according to the application of Super Simple Phosphate.

\begin{tabular}{ccc}
\hline Não Sensíveis & Sensíveis & Muito Sensíveis \\
$\Delta \mathrm{Qr}<10 \%$ e C.V. $<5,5 \%$ & $\Delta \mathrm{Qr}<20 \%$ e C.V. $<15 \%$ & $\Delta \mathrm{Qr}>20 \%$ e C.V. $>15 \%$ \\
\hline
\end{tabular}

$\Delta \mathrm{Qr}$ - variação da vazão relativa (\%); C.V. - coeficiente de variação (\%).

Nos casos em que a Qr e o C.V. não se enquadrarem no mesmo grupo, escolher o grupo baseado nos valores mais altos ou de C.V. ou de Qr.

Com relação à sensibilidade ao superfosfato simples, os emissores T4, T5, T6 T10, T14 e T20 foram classificados como "não sensíveis"; os emissores T2, T11, T12, T17, T18, T25 e T26 como "sensíveis" e os emissores T1, T3, T7, T8, T9, T13, T15, T16, T19, T21, T22, T23 e T24 como "muito sensíveis".

$\mathrm{Na}$ análise estatística dos emissores, em função da aplicação de superfosfato simples (Tabela 5), foi feita apenas a comparação entre os emissores da mesma linha. Estatisticamente, dos 26 emissores, 11 não sofreram influência da aplicação de superfosfato simples (não significativos) durante todo o experimento (T7, T9, T11, T12, T13, T16, T17, T19, T21, T22 e T24) e outros sete, que apesar de terem suas médias diferentes estatisticamente, mostraram que suas médias coletadas no momento inicial e ao final do experimento $(360 \mathrm{~h})$ foram iguais (mesma letra): T3, T4, T5, T10, T14, T15, T18. Os resultados são conflitantes com os comentados anteriormente, em que, por exemplo, o T24 que demonstrou alta sensibilidade ao superfosfato simples (C.V. $=110 \%)$; por meio dessa análise estatística, mostrou-se que os dados de vazão do T24 não diferiram significativamente, assim não sendo sensível ao superfosfato simples. Isso ocorre porque a análise estatística utilizada nessa abordagem foi baseada na média da vazão coletada, desconsiderando o coeficiente de variação que ocorre na leitura, já que os emissores não tendem a aumentar ou a diminuir a vazão no mesmo intervalo de tempo. Sendo, portanto, o C.V. e o Qr os parâmetros utilizados para dar conclusões a este artigo.

A Figura 2 foi confeccionada para avaliar a aplicação do ácido nítrico no controle do entupimento por supersimples, estando demonstrados os valores do grau de entupimento (GE) dos tratamentos antes e depois da aplicação do ácido. Percebe-se que a aplicação do ácido teve efeito negativo em 15 dos tratamentos, e apenas o emissor T25 não demonstrou sensibilidade ao ácido. Os emissores que foram beneficiados pela aplicação do ácido foram: T1, T2, T4, T14, T18, T19, T20, T21; entretanto, só os emissores T1 e T2 apresentam GE menor que 2\%. 
TABELA 5. Análise estatística das médias das vazões $\left(\mathrm{L} \mathrm{h}^{-1}\right)$ dos emissores em função da concentração, e do tempo de aplicação. Statistical analysis of the emitters average flow $\left(\mathbf{L ~ h}^{-1}\right)$.

\begin{tabular}{|c|c|c|c|c|c|c|c|c|c|}
\hline \multirow{2}{*}{ Emissor } & \multirow{2}{*}{ Q inicial } & \multicolumn{2}{|c|}{$50 \%$} & \multicolumn{2}{|c|}{$75 \%$} & \multicolumn{4}{|c|}{$100 \%$} \\
\hline & & $36 \mathrm{~h}$ & $72 \mathrm{~h}$ & $108 \mathrm{~h}$ & $144 \mathrm{~h}$ & $180 \mathrm{~h}$ & $216 \mathrm{~h}$ & $288 \mathrm{~h}$ & $360 \mathrm{~h}$ \\
\hline$\Gamma 1^{*}$ & $1,128 \mathrm{a}$ & $0,829 \mathrm{abc}$ & $0,618 \mathrm{~cd}$ & $0,925 \mathrm{ab}$ & $626 \mathrm{~cd}$ & ,636bcd & $0,644 \mathrm{bcd}$ & $0,367 \mathrm{de}$ & $0,234 \mathrm{e}$ \\
\hline$\Gamma 2 *$ & $1,910 b c$ & $2,22 \mathrm{a}$ & $1,895 b c$ & $2,024 a b$ & $1,955 b$ & $1,992 b$ & $2,028 \mathrm{ab}$ & $0,3667 \mathrm{de}$ & $0,2341 \mathrm{e}$ \\
\hline T3* & $2,022 \mathrm{ab}$ & $2,122 \mathrm{a}$ & 1,910ab & $2,204 a$ & $1,918 \mathrm{ab}$ & $2,073 \mathrm{ab}$ & $1,999 \mathrm{ab}$ & $1,894 \mathrm{ab}$ & $1,720 \mathrm{~b}$ \\
\hline $\mathrm{T} 4 *$ & $3,852 \mathrm{c}$ & $4,192 \mathrm{ab}$ & $4,010 \mathrm{abc}$ & $4,06583 \mathrm{abc}$ & $3,960 \mathrm{abc}$ & $4,240 \mathrm{a}$ & $3,877 \mathrm{c}$ & $3,909 \mathrm{bc}$ & $3,977 \mathrm{abc}$ \\
\hline T5* & $2,695 \mathrm{dc}$ & $2,744 \mathrm{bcd}$ & $2,677 d$ & $2,840 \mathrm{a}$ & $2,717 \mathrm{acd}$ & $2,788 \mathrm{ab}$ & $2,845 \mathrm{a}$ & $2,800 \mathrm{ab}$ & $2,776 a b c$ \\
\hline $66^{*}$ & $2,011 \mathrm{~b}$ & $2,344 a b$ & $2,388 \mathrm{ab}$ & $2,375 \mathrm{ab}$ & $2,478 \mathrm{a}$ & $2,453 a$ & $2,434 \mathrm{a}$ & 2,392ab & $2,459 a$ \\
\hline $7 \mathrm{~ns}$ & $2,024 \mathrm{a}$ & $2,292 \mathrm{a}$ & $2,031 \mathrm{a}$ & $2,289 \mathrm{a}$ & $2,247 \mathrm{a}$ & $2,295 \mathrm{a}$ & $045 \mathrm{a}$ & $2,184 a$ & $1,992 \mathrm{a}$ \\
\hline$\Gamma 8^{*}$ & $2,047 \mathrm{a}$ & $1,902 \mathrm{abcd}$ & $1,939 \mathrm{abc}$ & 2,009ab & $1,745 \mathrm{~cd}$ & 1,909abcd & $1,978 \mathrm{abc}$ & $1,788 \mathrm{bcd}$ & $1,675 d$ \\
\hline$\Gamma 9 \mathrm{~ns}$ & $1,945 \mathrm{a}$ & $1,974 \mathrm{a}$ & $1,984 a$ & $1,922 \mathrm{a}$ & $2,010 \mathrm{a}$ & $1,947 \mathrm{a}$ & $1,970 \mathrm{a}$ & $1,845 \mathrm{a}$ & $1,799 \mathrm{a}$ \\
\hline$\Gamma 10 *$ & 2,293ab & $2,234 \mathrm{ab}$ & $2,258 \mathrm{ab}$ & $2,317 \mathrm{ab}$ & $2,341 \mathrm{a}$ & 2,279ab & $2,268 \mathrm{ab}$ & $2,333 \mathrm{ab}$ & $2,308 \mathrm{ab}$ \\
\hline $11 \mathrm{~ns}$ & $854 a$ & $2,080 \mathrm{a}$ & $1,988 \mathrm{a}$ & $2,171 \mathrm{a}$ & $1,929 a$ & $1,995 \mathrm{a}$ & $2,065 \mathrm{a}$ & $1,911 \mathrm{a}$ & $1,898 \mathrm{a}$ \\
\hline $12 \mathrm{~ns}$ & ,136a & $1,966 \mathrm{a}$ & $2,017 \mathrm{a}$ & $1,919 \mathrm{a}$ & $1,917 \mathrm{a}$ & $1,818 \mathrm{a}$ & $150 \mathrm{a}$ & $2,055 \mathrm{a}$ & $2,027 \mathrm{a}$ \\
\hline $13 \mathrm{~ns}$ & $2,477 \mathrm{a}$ & $2,345 \mathrm{a}$ & $2,298 \mathrm{a}$ & $2,589 \mathrm{a}$ & $2,318 \mathrm{a}$ & $2,137 \mathrm{a}$ & $2,292 \mathrm{a}$ & $2,121 \mathrm{a}$ & $2,135 \mathrm{a}$ \\
\hline$\Gamma 14 *$ & $2,302 \mathrm{ab}$ & $2,230 \mathrm{abc}$ & $2,256 a b c$ & $2,193 \mathrm{cb}$ & $2,249 \mathrm{abc}$ & $2,146 \mathrm{c}$ & $2,346 a$ & $2,305 \mathrm{ab}$ & $2,277 \mathrm{ab}$ \\
\hline T15* & $1,746 a b$ & $1,726 a b$ & $1,990 \mathrm{a}$ & $1,823 \mathrm{ab}$ & $1,736 a b$ & $1,715 \mathrm{ab}$ & $1,687 b$ & 1,549 & $1,549 \mathrm{~b}$ \\
\hline T16 ns & $2,874 a$ & $2,258 \mathrm{a}$ & $2,199 \mathrm{a}$ & $2,614 a$ & $2,453 a$ & $2,361 \mathrm{a}$ & $2,352 \mathrm{a}$ & $2,440 \mathrm{a}$ & $2,736 a$ \\
\hline $\mathrm{T} 17 \mathrm{~ns}$ & $2,5107 \mathrm{a}$ & $2,631 \mathrm{a}$ & $2,433 a$ & $2,653 a$ & $2,394 a$ & $2,739 a$ & $2,5938 \mathrm{a}$ & $2,323 a$ & $2,485 a$ \\
\hline $18 *$ & ,085ab & $2,112 \mathrm{ab}$ & $2,129 a b$ & $2,132 \mathrm{ab}$ & $2,115 \mathrm{ab}$ & $1,974 b$ & $2,173 \mathrm{ab}$ & $2,156 a b$ & $2,231 \mathrm{a}$ \\
\hline $19 \mathrm{~ns}$ & $1,501 \mathrm{a}$ & $1,550 \mathrm{a}$ & $1,648 \mathrm{a}$ & $1,658 \mathrm{a}$ & $1,485 \mathrm{a}$ & $1,653 \mathrm{a}$ & $1,574 \mathrm{a}$ & $1,633 \mathrm{a}$ & $1,630 \mathrm{a}$ \\
\hline$\Gamma 20 *$ & $2,528 \mathrm{a}$ & $2,445 \mathrm{ab}$ & $2,361 b$ & $2,470 \mathrm{a}$ & $2,432 \mathrm{ab}$ & $2,421 \mathrm{ab}$ & $2,405 \mathrm{ab}$ & $2,090 \mathrm{c}$ & $2,186 c$ \\
\hline $\mathrm{T} 21 \mathrm{~ns}$ & $1,744 \mathrm{a}$ & $1,721 \mathrm{a}$ & $1,570 \mathrm{a}$ & $1,732 \mathrm{a}$ & $1,592 \mathrm{a}$ & $1,622 \mathrm{a}$ & $1,527 \mathrm{a}$ & $1,949 \mathrm{a}$ & $1,499 a$ \\
\hline T22 ns & $2,238 \mathrm{a}$ & $2,346 \mathrm{a}$ & $2,090 \mathrm{a}$ & $2,423 a$ & $2,106 \mathrm{a}$ & $2,321 \mathrm{a}$ & $2,213 \mathrm{a}$ & $2,0612 \mathrm{a}$ & $2,079 \mathrm{a}$ \\
\hline T23* & $1,202 \mathrm{a}$ & $1,2596 \mathrm{a}$ & $1,360 \mathrm{a}$ & $1,19194 \mathrm{a}$ & $1,118 \mathrm{a}$ & $1,245 \mathrm{a}$ & $1,128 \mathrm{a}$ & $1,138 \mathrm{a}$ & $0,711 b$ \\
\hline $\mathrm{T} 24 \mathrm{~ns}$ & $0,475 a$ & $0,701 \mathrm{a}$ & $1,235 \mathrm{a}$ & $0,995 \mathrm{a}$ & $0,363 \mathrm{a}$ & $0,428 \mathrm{a}$ & $0,861 \mathrm{a}$ & $0,708 \mathrm{a}$ & $0,378 \mathrm{a}$ \\
\hline T) & $1,624 d$ & $1,718 \mathrm{bcd}$ & $1,668 d \mathrm{~d}$ & $1,790 a b c$ & $1,824 \mathrm{ab}$ & $1,859 \mathrm{a}$ & $1,773 a b c$ & $1,893 \mathrm{a}$ & $1,816 \mathrm{ab}$ \\
\hline 26 & $1,530 \mathrm{e}$ & $1,531 \mathrm{e}$ & $558 \mathrm{de}$ & $1,650 \mathrm{~cd}$ & $1,677 b c$ & $1,620 \mathrm{cde}$ & $1,768 \mathrm{ab}$ & $1,827 \mathrm{a}$ & 1,772 \\
\hline
\end{tabular}

* Comparação de médias que foram significativos a $1 \%$; ${ }^{\text {ns }}$ Comparação de médias não significativas. Médias seguidas de mesma letra na linha não diferem entre si.

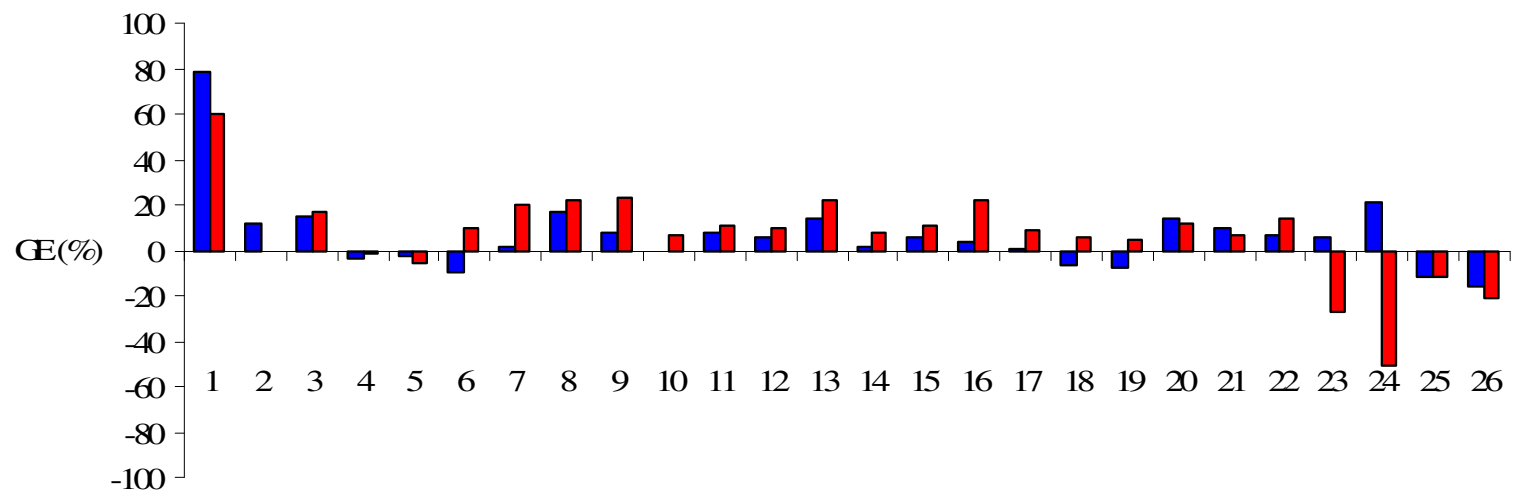

Tratamentos
— Antes da aplicação de Ácido Nítrico $\square$ Após a aplicação de Ácido Nítrico

FIGURA 2. Grau de entupimento dos emissores antes e após a aplicação do ácido nítrico (os valores negativos refletem aumento da vazão). Clogging degree of the emitters before and after the application of nitric acid (negative values reflect increased flow). 
O ácido não conseguiu desentupir os gotejadores da linha dos emissores T9, T16, T21 e T24, (Figura 3), entretanto conseguiu reverter o quadro nos gotejadores da linha do emissor T23, e provocou o entupimento de mais dois gotejadores na linha dos emissores no T7 e T9. Um dos motivos pela falta de eficiência seria o desprendimento de substâncias das paredes da tubulação, ocasionadas pelo ácido, e essas ter-se-iam concentrado nas saídas dos emissores, aumentando o grau de entupimento (GE).
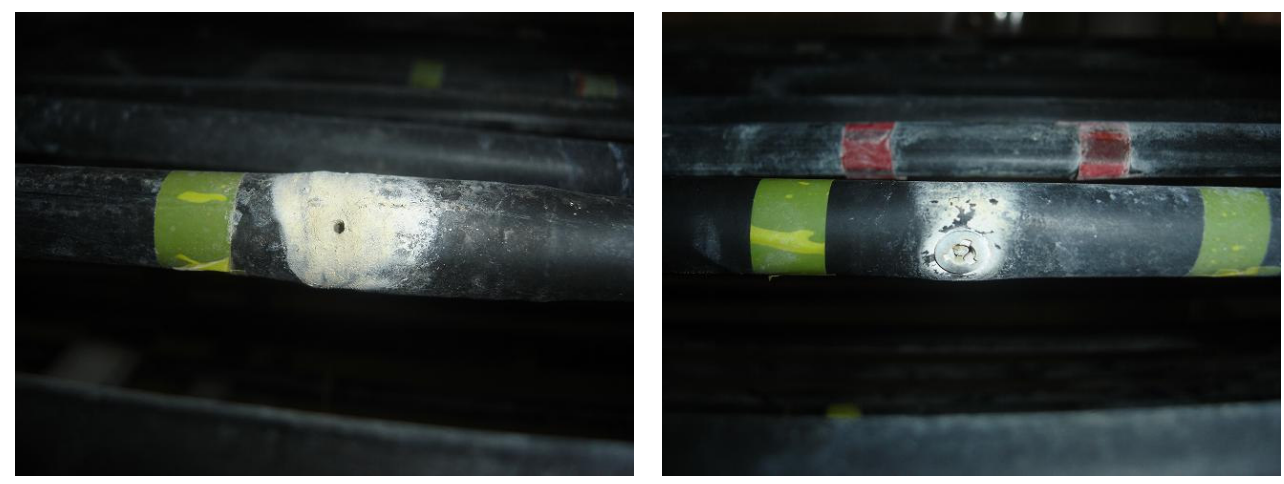

FIGURA 3. Foto de gotejadores que sofreram entupimento total, T9 (direita) e T16 (esquerda), ao final da aplicação do ácido nítrico. Photo of clogged drippers, T9 (right) and T16 (left), after the application of nitric acid.

\section{CONCLUSÕES}

O entupimento parcial foi em maior número que o entupimento total.

Não é possível relacionar o entupimento com o tipo de fluxo do emissor (convencional e autocompensante).

O T24 demonstrou maior suscetibilidade à aplicação do superfosfato simples (SS) - C.V. igual a $110 \%$.

O ácido nítrico, aplicado segundo esta metodologia, não proporcionou a desobstrução dos emissores, tendo efeito contrário, induzindo o entupimento dos mesmos.

\section{REFERÊNCIAS}

ANUÁRIO ESTATÍSTICO. São Paulo: ANDA, 2004. 2v.

BRITO, L.T. de L.; COSTA, N.D.; SOARES J.M.; FARIA, C.M.B. Fontes de fósforo aplicadas via fertirrigação na cultura do melão. In: REUNIÃO BRASILEIRA DE FERTILIDADE DO SOLO E NUTRIÇÃO DE PLANTAS, 22., 1996, Manaus. Resumos... Manaus:

SBCS/UA/EMBRAPA/INPE, 1996. p.194.

BRITO, L.T. de L.; SOARES, J.M.; FARIA, C.M.B.; COSTA, N.D. Fontes de fósforo aplicadas na cultura do melão via água de irrigação. Revista Brasileira de Engenharia Agrícola e Ambiental, Campina Grande, v.4, n.1, p.19-22, 2000.

FAGERIA, N.K. Resposta de arroz de terras altas à correção de acidez em solo de cerrado. Pesquisa Agropecuária Brasileira, Brasília, v.35, n.11, p.2.303-2.307, nov. 2000.

FORATTO, L.C.; ZANINI, J.R.; NATALE, W. Teor de fósforo e pH no bulbo molhado, com diferentes frequências de fertirrigação, utilizando ácido fosfórico. Engenharia Agrícola, Jaboticabal, v.27, n.2, p.436-444, 2007.

FRANSON, M.A.H. Standard methods for the examination of water and wastewater. Washington: American Public Health Association, 1995. 1.074 p. 
MEDEIROS, P.R.F.; COELHO, R.D.; BARROS, A.C.; MELO, R.F. Dinâmica do entupimento de gotejadores em função da aplicação de carbonato de potássio. Irriga, Botucatu, v.13, n.2, p.288297, 2008.

NAKAYAMA, F.S.; BUCKS, D.A. Trickle irrigation of crop: production design, operation and management. Amsterdam: Elsevier, 1986. 383 p.

RETTORE NETO, O. Metodologia para determinação da perda de carga localizada em emissores não coaxiais integrados a tubos de polietileno. 2008. 62 f. Dissertação (Mestrado em Irrigação e Drenagem) - Escola Superior de Agricultura "Luiz de Queiroz", Universidade de São Paulo, Piracicaba, 2008.

TEIXEIRA, M.B. Efeitos de dosagens extremas de cloro e pH na vazão de gotejadores autocompensantes (irrigação localizada). 2006. 322 f. Tese (Doutorado em Irrigação e Drenagem) - Escola Superior de Agricultura “Luiz de Queiroz”, Universidade de São Paulo, Piracicaba, 2006.

VILLAS BÔAS, R.L.; BÜLL, L.T.; FERNÁNDEZ, D.M. Fertilizantes em fertirrigação. In: FOLEGATTI, M.V. (Coord.). Fertirrigação: cítrus, flores e hortaliças. Guaiba: Agropecuária, 1999. p.293-319.

VITI, G.C.; BOARETTO, A.E. Fertilizantes fluidos. Piracicaba: POTAFÓS, 1994. p.261-281.

VITTI, G.C.; BOARETTO, A.E.; PENTEADO, S.R. Fontes de fertilizantes e fertirrigação. In: SIMPÓSIO BRASILEIRO SOBRE FERTILIZANTES FLUIDOS. Piracicaba, 1993. Anais... Piracicaba: ESALQ/CENA, POTAFOS, 1994. p.233-256. 